\title{
Hochempfindliches Nachweisverfahren auf der Basis der Fluorescenz durch Laser-Anregung
}

\author{
Claude Aubert ${ }^{1}$, Jürg Fünfschilling ${ }^{1}$, Iris Zschokke-Gränacher ${ }^{1}$ und Heinz Langhals ${ }^{2}$ \\ 1 Institut für Physik der Universität Basel, Klingelbergstraße 82, CH-4056 Basel, Schweiz \\ 2 Institut für Organische Chemie der Universität München, Karlstraße 23, D-8000 München 2, \\ Bundesrepublik Deutschland
}

\section{Highly Sensitive and Selective Method for Analysis by Use of Laser Excited Fluorescence}

Summary. Due to their high photostability and fluorescence quantum yield perylene dyes prove to be ideal labels for fluorescence measurements at extremely low concentrations and high excitation intensities. In solid solutions at $10 \mathrm{~K}$ a detection sensitivity of up to 1 dye molecule in $10^{14}$ solvent molecules $\left(4 \cdot 10^{-18} \mathrm{~mol}\right)$ is reached. Calibration with the Raman emission of the used matrix rises the precision of the analysis to $5-10 \%$ at this dilution. Modification of the perylene dyes allows labelling in aqueous and organic phases. Applications in biochemistry are discussed.

Zusammenfassung. Dank ihrer extrem hohen Photostabilität und hohen Fluorescenzquantenausbeute erwiesen sich die Perylenfarbstoffe als ideal für Markierungen bei sehr kleinen Konzentrationen und hohen Anregungsintensitäten. In fester Matrix wird bei $10 \mathrm{~K}$ eine Nachweisempfindlichkeit von 1 Farbstoffmolekül in $10^{14}$ Solvensmolekülen erreicht $\left(4 \cdot 10^{-18} \mathrm{~mol}\right)$. Durch eine Eichung mit den Raman-Linien der verwendeten Matrices läßt sich bei dieser Verdünnung eine Analysengenauigkeit von $5-10 \%$ erreichen. Durch $\mathrm{Ab}$ wandlung der Perylenfarbstoffe sind Markierungen in wäßriger und organischer Phase möglich. Anwendungen in der Biochemie werden aufgezeigt.

\section{Einleitung}

Werden bei analytischen Nachweisverfahren hohe Empfindlichkeiten benötigt, so setzt man z.Zt. üblicherweise radioaktive Markierungen ein. Noch niedrigere Nachweisgrenzen sollten aber mit der optischen Fluorescenz zu erreichen sein. Während bei der ersteren Methode markierte Moleküle nur einmal zerfallen können, sind bei Fluorescenzfarbstoffen mit hoher Photostabilität bis zu $10^{9}$ Anregungsund Fluorescenzschritte möglich, bevor ein Photobleichprozeß erfolgt [1]. Darüber hinaus bieten die Fluorescenzfarbstoffe den Vorteil der gefahrlosen Handhabung und geringeren Störungen empfindlicher Systeme, da in den zu untersuchenden Proben keine ionisierenden Strahlen freigesetzt werden.

\section{Ergebnisse}

Wie in vorangegangenen Arbeiten berichtet wurde [1-3], weisen die Perylenfarbstoffe 1 extrem hohe Photostabilitäten und Fluorescenzquantenausbeuten $(\Phi)$ bis zu $100 \%$ auf. Eine gute Löslichkeit in organischer Matrix wird durch die Substitution mit tert-Butylgruppen erreicht $[1,2,4]$, so z.B. bei 1 a mit $\Phi=99 \%$. Darüber hinaus werden die UV/ VIS-Absorptions- und Fluorescenzspektren von 1 nur wenig vom Solvens und dem Substituenten $R$ beeinflußt. Zudem liegt die Absorption und Fluorescenz in einem spektralen Bereich ( 525 bzw. $540 \mathrm{~nm}$ ), in dem die üblichen organischen Solventien, sowie der überwiegende Teil der organischen und biochemischen Substrate keine Absorption aufweisen.

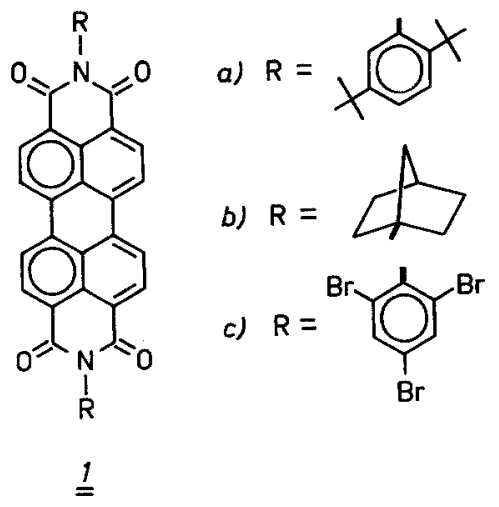

Eine hohe Nachweisempfindlichkeit für Farbstoff 1 wird durch die Fluorescenzmessung unter Laser-Anregung unterhalb $10 \mathrm{~K}$ erreicht. Unter diesen Bedingungen werden charakteristische Linienspektren erhalten (sog. Site-Selection-Spektren [5, 6]; Abb. 1), die sich deutlich von dem übrigen Fluorescenzuntergrund abheben. Die geringe Halbwertsbreite der Fluorescenzlinien, deren Lage zudem für den jeweiligen Farbstoff charakteristisch ist, gewährleistet ein hohes Signal-zu-Rausch-Verhältnis und damit eine hohe Empfindlichkeit. Wesentlich ist dabei die hervorragende Photostábilität der Farbstoffe 1, bei welchen ein Photobleichen während der Meßzeit nur wenige Prozent erreicht.

Die Photostabilität der Farbstoffe 1 ist allerdings vom verwendeten Medium abhängig. Ether erweisen sich hier als besonders günstig, zum einen in bezug auf die Photostabilität der gelösten Farbstoffe, und zum anderen bilden sie bei tiefer Temperatur ein für die Messung gut geeignetes Glas. Die drei hier näher untersuchten Perylenfarbstoffe 1a ( $\mathrm{R}=2,5$-Di-tert-butylphenyl), 1 b (1-Norbornyl) und 1c 


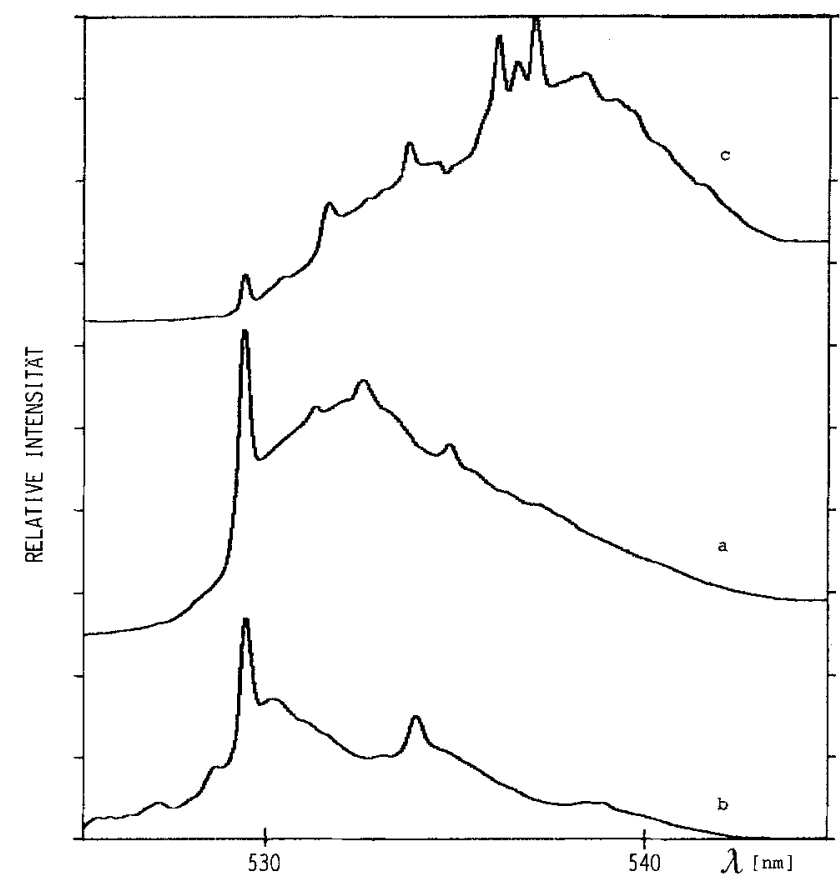

Abb. 1. Site-Selection-Spektren der Perylenfarbstoffe 1a (Konz. $\left.1,71 \cdot 10^{-7} \mathrm{~mol} \cdot 1^{-1}\right), 1 \mathbf{b}\left(4,23 \cdot 10^{-7} \mathrm{~mol} \cdot 1^{-1}\right)$ und $1 \mathrm{c}(4,67$ $\cdot 10^{-7} \mathrm{~mol} \cdot \mathrm{l}^{-1}$ ) bei $10 \mathrm{~K}$ (Anregung bei $488,0 \mathrm{~nm}$ )

(2,4,6-Tribromphenyl) zeigen in 2-Methyltetrahydrofuran (MTHF) bzw. Diethylether bei $10 \mathrm{~K}$ und bei der von uns verwendeten Emissionslinie ein ähnliches Ausbleichverhalten. Bei Zimmertemperatur hingegen sind die beobachteten Unterschiede in den Ausbleichraten ausgeprägter [2].

Das hier geschilderte Verfahren basiert auf einer Absolutmessung der Fluorescenzintensität. Eine weitere, wesentliche Verbesserung bringt eine Eichung der Fluorescenzintensität mit den Raman-Linien des Solvens, deren Intensität in der gleichen Größenordnung liegt wie die Intensität der Fluorescenzlinien bei verdünnten Proben [7]. Durch diese Relativmessung wird eine wesentliche Steigerung der Genauigkeit und Verläßlichkeit erreicht. Über die interne Eichung an derselben Probe werden Fehlerquellen, die beispielsweise auf die Geometrie des Strahlengangs zurückzuführen sind, vermieden.

Auf diesem Wege wird für 1 a in MTHF bzw. Diethylether eine Nachweisempfindlichkeit von $10^{-13} \mathrm{~mol} \cdot \mathrm{l}^{-1}$ bzw. $7 \cdot 10^{-14} \mathrm{~mol} \cdot 1^{-1}$ bei einer Analysengenauigkeit von ca. $5-10 \%$ erreicht. Berücksichtigt man dabei ein minimales Analysenvolumen von ca. $0,05 \mathrm{ml}$, so lassen sich mit der genannten Genauigkeit noch $4 \cdot 10^{-18} \mathrm{~mol}$ der Farbstoffe nachweisen. Die genannte Nachweisgrenze wird hauptsächlich durch Unsicherheiten bei der Bestimmung der Fluorescenzintensität festgelegt. Die Ursachen sind Drift-Vorgänge, die Photonenstatistik und auch das Ausbleichen der Farbstoffe.

Um letzteren Einfluß quantitativ zu erfassen, wird im folgenden das Ausbleichverhalten der Perylenfarbstoffe in verschiedenen Medien untersucht. Es zeigt sich dabei, daß die Perylenfarbstoffe in Ethern wie MTHF oder Diethylether sehr photostabil sind, so daß innerhalb der Meßzeit auch bei stärksten Verdünnungen nur wenige Prozent ausbleichen, während die Photostabilität in Alkoholen, wie z. B. Ethanol deutlich geringer ist ( 25 bis $50 \%$ bleichen innerhalb der
Meßzeit aus). Der Photobleichprozeß ist jedoch reversibel und kann durch Erwärmen der Probe ausgeheilt werden [7]. Über weitere Einzelheiten dieses Prozesses wird an anderer Stelle berichtet werden. Als geeignetes Medium für analytische Arbeiten erweist sich ferner Silicongummi, welcher eine Ausbleichrate von ca. 0,2\% innerhalb der Meßzeit aufweist und außerdem den weiteren Vorteil hat, bei Zimmertemperatur fest zu sein. Ein Probengläschen ist hier somit für die Messung nicht erforderlich.

$\mathrm{Da}$ bei Laser-Anregung die Fluorescenz-Emission scharfe Linien aufweist, deren Lage von der Substitution der Farbstoffe abhängt, ist es auch möglich, in Proben mit mehreren Perylenfarbstoffen, diese simultan zu bestimmen. In MTHF gelingt dies z.B. bei den Farbstoffen $1 \mathbf{a}$ und $\mathbf{1 c}$ bei einem Mischungsverhältnis von 100:1 bis 1:100 mit jeweils einem Fehler von weniger als $10 \%$. Optische Inhomogenitäten und Verunreinigungen in der Probe haben dabei nur einen geringen Einfluß auf das Analysenergebnis.

In organischen Medien können die Farbstoffe 1 direkt eingesetzt werden, in Wasser sind sie jedoch schwerlöslich. In wäßriger Phase wird besser die Sulfonsäure 2 oder in saurem Medium auch das Diamin 3 [3] verwendet. Für Markierungen ist weiterhin das Anhydrid-Imid $4(X=0)$ [3] wichtig, das z.B. für biochemische Anwendungen mit primären Aminen $\left(\mathrm{R}^{\prime}-\mathrm{NH}_{2}\right)$ zu Perylenfarbstoffen $\left(X=R^{\prime}-N\right)$ kondensiert werden kann, die noch eine freie Aminogruppe tragen. Über die zweite Amino-Funktion wird dabei eine genügende Wasserlöslichkeit erreicht. Auf diesem Wege sind Markierungen mit fluorescierenden Perylen-Systemen möglich.
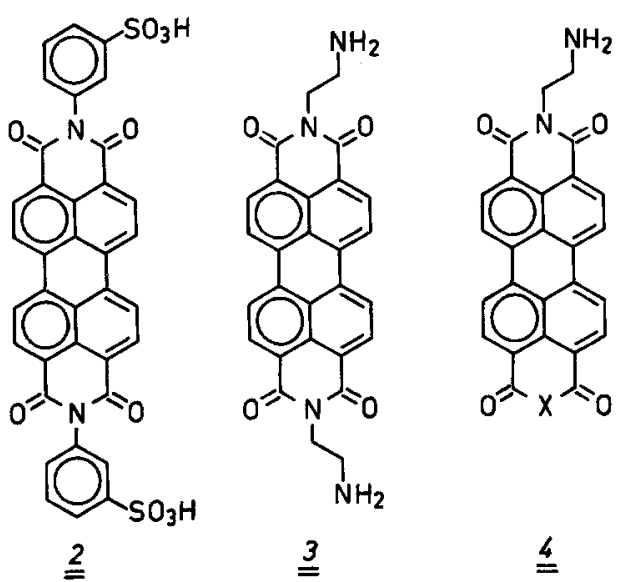

\section{Experimenteller Teil}

$N, N^{\prime}$-Bis(phenyl-3-sulfonsäure)-3,4:9, 10-perylenbiscarboximid 2 [8]

$1,0 \mathrm{~g}(1,34 \mathrm{mmol}) \mathrm{N}, \mathrm{N}^{\prime}$-Bis(3-sulfonamidophenyl)-3,4:9,10perylenbis-carboximid ([2]; R 24) werden mit $40 \mathrm{ml}$ konz. Schwefelsäure auf $60^{\circ} \mathrm{C}$ erwärmt, danach unter Rühren mit $300 \mathrm{mg}(4,3 \mathrm{mmol})$ Natriumnitrit versetzt und $2 \mathrm{~h}$ auf $80^{\circ} \mathrm{C}$ erwärmt. Die Reaktionslösung wird auf Eis gegossen und die Sulfonsäure durch vorsichtige Zugabe von Schwefelsäure gefällt. Die stark hygroskopische Sulfonsäure wird abzentrifugiert, mit Wasser gewaschen und zur weiteren Reinigung in $500 \mathrm{ml}$ heißem Wasser gelöst und durch Zugabe von Calciumchloridlösung als Calciumsalz gefällt. Ausbeute Rohprodukt: $2,6 \mathrm{~g}$; zur weiteren Reinigung kann das Calciumsalz aus Wasser umkristallisiert werden. 
IR $(\mathrm{KBr}): 1695,1660(\mathrm{C}=0), 1200 \mathrm{~cm}^{-1}\left(\mathrm{SO}_{2}\right)$.

Das Natriumsalz wird durch Zugabe einer ges. Lösung von Natriumsulfat zu einer wäßrigen Lösung des Calciumsalzes und Umkristallisation aus Wasser erhalten.

In Mischungen von Wasser mit organischen Lösungsmitteln beträgt die Fluorescenzquantenausbeute der Sulfonsäure bzw. des Natriumsalzes $80 \%$ bis zu einem Wassergehalt von ca. 60 Vol. \%. Bei höherem Wassergehalt der Lösung fällt dann die Fluorescenzquantenausbeute des Farbstoffs, vermutlich wegen Assoziation, stark ab und beträgt in reinem Wasser noch ca. 10\%.

\section{Meßanordnung [7]}

Zur Konzentrationsbestimmung wird als Lichtquelle ein lichtstabilisierter Argonlaser verwendet, dessen Lichtleistung mit Hilfe von Filtern zwischen 0,5 und $100 \%$ $(200 \mathrm{~mW})$ eingestellt werden kann. Über ein Prisma werden die Plasmalinien des Lasers unterdrückt, und der Laserstrahl wird dann auf die feste Lösung fokussiert, welche in einem Helium-Durchfluß-Kryostat (Fa. Oxford Instruments) auf eine Temperatur von $10 \mathrm{~K}$ thermostatisiert wird. Das Probenvolumen kann problemlos bis auf $0,05 \mathrm{ml}$ reduziert werden. Für Routineuntersuchungen empfiehlt es sich aber wegen einer einfacheren Probeneinfüllung ein Volumen von $0,5 \mathrm{ml} \mathrm{zu}$ verwenden. Das Fluorescenzlicht der Probe wird auf den Eintrittsspalt ( $100 \mu \mathrm{m} \cong 0,16 \mathrm{~nm}$ Auflösung) eines $0,50 \mathrm{~m}$ Spektrometers fokussiert. Mit Hilfe eines Kantenfilters wird das Streulicht des Lasers unterdrückt. Das Gitter des Spektrometers (1200 Linien/mm) wird über einen Stepmotor von einem Computer gesteuert. Die Detektion des Lichtes erfolgt mit einem sog. optischen Multichannel Analyser (OMA, PAR Modell 1254 der Fa. EG \& G), dessen Videokamera gekühlt wird. Durch die Kühlung wird der Dunkelstrom des Detektors um einen Faktor 100 erniedrigt und dadurch eine für die Messung wesentliche Steigerung des Signal-zu-Rausch-Verhältnisses erreicht. Die Abtastung des elektrischen OMA-Ausgangssignals erfolgt durch den bereits erwähnten Computer (APPLE II +), der außerdem eine mechanische Klappe steuert, die den Strahlengang des Anregungslasers nur während des Meßvorgangs freigibt. Dies ist wichtig, um ein unkontrolliertes Ausbleichen der Probe zu vermeiden.

Die Testlösungen wurden aus einer Stammlösung mit einer Konzentration von $10^{-6} \mathrm{~mol} \cdot 1^{-1}$ in höchstens drei Verdünnungsschritten hergestellt, um den Fehler in der Konzentration möglichst klein zu halten $(<3 \%)$. Wegen der großen Empfindlichkeit der Meßmethode wurden die Küvetten, Meßkolben usw. nur einmal verwendet.

Für die Ausführung der Analyse werden folgende Messungen benötigt:

1. Das Fluorescenzspektrum (Site-Selection-Spektrum) der Probe,

2. Das Raman-Spektrum der Probe,

3. Das Spektrum der reinen Matrix.

Als Referenz wird weiterhin von der Apparatur ein Dunkelstromspektrum und zur Eichung der spektralen Empfindlichkeit der Apparatur das Spektrum einer Wolfram-Halogenlampe aufgenommen. Zur Auswertung wird das Site-Selection-Spektrum der Lösung und das Spektrum der reinen Matrix in bezug auf den Untergrund und das kontinuierliche Spektrum der Wolfram-Halogenlampe korrigiert. Von der Fläche der verwendeten Linie des Site-Selection-Spektrums wird in diesem Wellenlängenbereich das

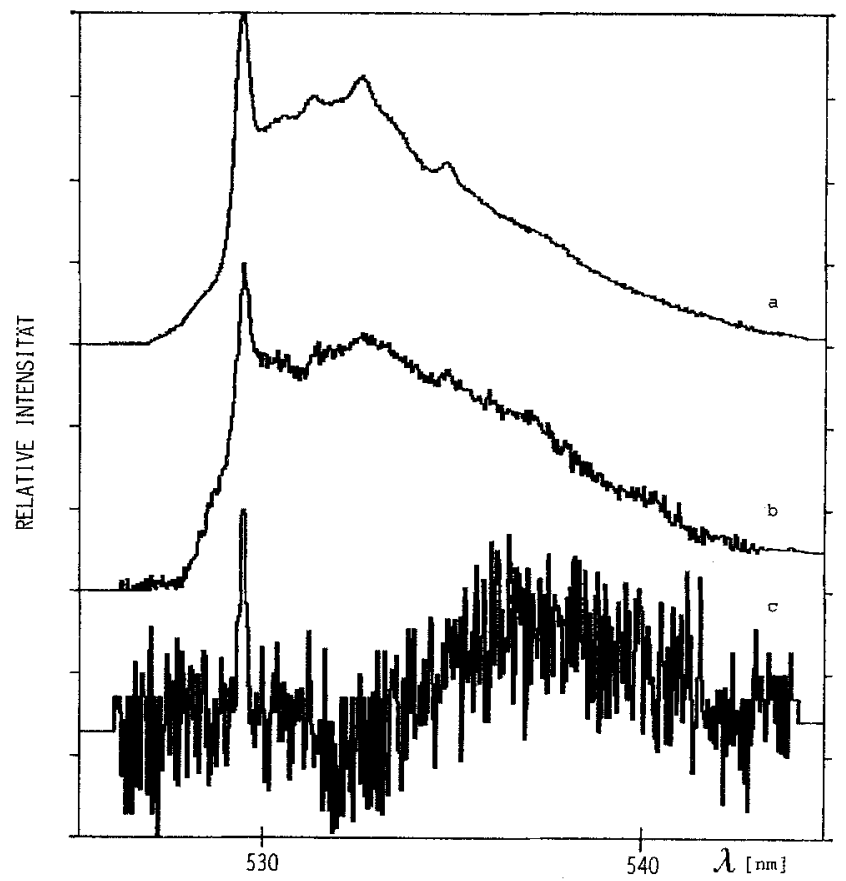

Abb. 2. Site-Selection-Spektren des Perylenfarbstoffs 1 a in MTHF (Anregung bei $488.0 \mathrm{~nm} ; 10 \mathrm{~K}$ ); a $8,82 \cdot 10^{-11} \mathrm{~mol} \cdot 1^{-1}$; b $6,80 \cdot 10^{-12} \mathrm{~mol} \cdot 1^{-1 ;}$ c $2,5 \cdot 10^{-13} \mathrm{~mol} \cdot 1^{-1}$. Alle Spektren sind bezüglich Dunkelstrom, spektraler Empfindlichkeit der Apparatur und Fluorescenz des Lösungsmittels korrigiert

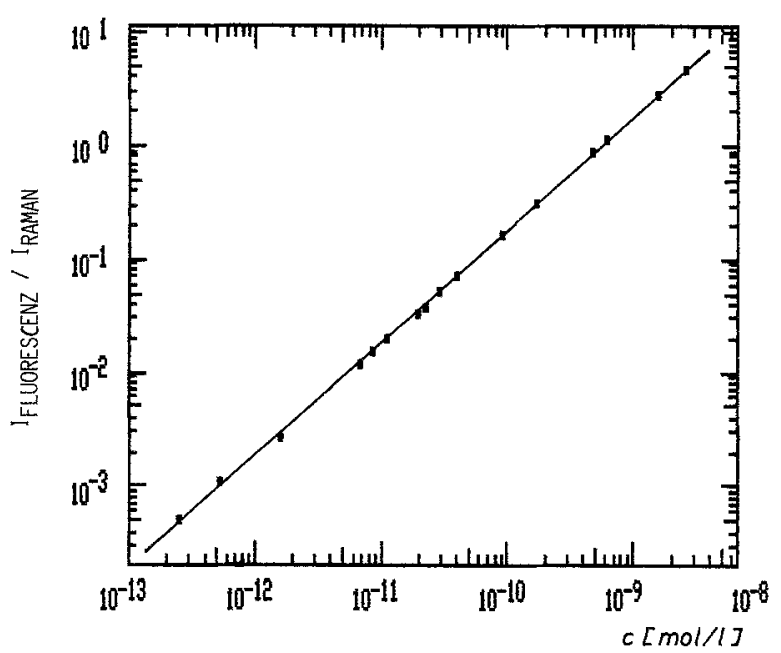

Abb. 3. Auf die Raman-Intensität der Matrix normierte Fluorescenzintensität ( $\left(\mathrm{I}_{\text {Fluorescenz }} / \mathrm{I}_{\text {Raman }}\right)$ als Funktion der Farbstoff-Konzentration. $\left(I_{\text {Fluorescenz }} / I_{\text {Raman }}=1,7727 \cdot 10^{9} \cdot \mathrm{c}\left[\mathrm{mol} \cdot \mathrm{I}^{-1}\right]\right)$ für $\mathbf{1} \mathbf{a}$ in MTHF

Spektrum der reinen Matrix subtrahiert. Die so erhaltene Fläche dieser Linie des Site-Selection-Spektrums wird auf die Fläche der Raman-Linien der Matrix normiert. Dieser Quotient ist ein direktes Maß für die gesuchte FarbstoffKonzentration. Abbildung 2 zeigt die korrigierten Spektren einer Konzentrationsreihe des Farbstoffs 1 a in MTHF [7].

Zur Konzentrationsbestimmung von 1 a wurde hier die Intensität der Fluorescenz-Linie des Site-Selection-Spektrums bei $529,5 \mathrm{~nm}$ verwendet, sowie bei der Matrix MTHF die Summe der drei Raman-Linien der C-H-Vibrationen bei 2861,2924 und $2968 \mathrm{~cm}^{-1}$. In Abb. 3 ist der genannte 
Quotient aus Fluorescenz-/Ramanintensität gegen die Farbstoff-Konzentration aufgetragen. Die Nachweisgrenze von ca. $10^{-13} \mathrm{~mol} \cdot 1^{-1}$ bei $5-10 \%$ Genauigkeit wird hauptsächlich durch Unsicherheiten bei der Bestimmung der Fluorescenzintensität festgelegt. Das Photobleichen des Farbstoffes spielt demgegenüber in dem genannten Medium nur eine untergeordnete Rolle.

Da die Site-Selection-Spektren charakteristische, scharfe Linien liefern, sind mit der hier vorgestellten Methode auch Analysen von Farbstoff-Mischungen oder verunreinigten Proben problemlos mit hoher Analysengenauigkeit möglich.

\section{Analysenbeispiel}

Aus $109 \mu \mathrm{g}$ Farbstoff 1 a wird durch Auffüllen auf $100 \mathrm{ml}$ mit MTHF eine Stammlösung bereitet (Gehalt: 1,42 $\left.\cdot 10^{-6} \mathrm{~mol} \cdot \mathrm{1}^{-1}\right)$. Hiervon ausgehend wird in drei Verdünnungsschritten $(7,78 \mu \mathrm{l}$ auf $0,35 \mathrm{ml}, 9,11 \mu \mathrm{l}$ auf $0,30 \mathrm{ml}$ und $3,90 \mu \mathrm{l}$ auf $0,55 \mathrm{ml}$ ) ein Gehalt von $6,80 \cdot 10^{-12} \mathrm{~mol} \cdot 1^{-1}$ erhalten. Von dieser Lösung wird eine Probe von $0,5 \mathrm{ml}$ zur Analyse eingesetzt (das Volumen kann problemlos bis auf $0,05 \mathrm{ml}$ reduziert werden - das entspricht einem absoluten Gehalt von $10^{-16} \mathrm{~mol}$ ) und ergibt ein Verhältnis $I_{\text {Fluorescenz }}$
$\mathrm{I}_{\text {Raman }}$ von $1,16 \cdot 10^{-2}$. Mit Hilfe der Eichgerade von Abb. 3 $\left(\mathrm{I}_{\text {Fluorescenz }} / \mathrm{I}_{\text {Raman }}=1,7727 \cdot 10^{9} \cdot \mathrm{c}\right)$ wird eine Konzentration von $6,54 \cdot 10^{-12} \mathrm{~mol} \cdot 1^{-1}$ erhalten. Die Abweichung vom eingewogenen Wert beträgt $3,8 \%$.

Der Stiftung Volkswagenwerk und dem Schweizer Nationalfonds zur Förderung der wissenschaftlichen Forschung wird für die Unterstützung der Arbeit gedankt, sowie Herrn Prof. Dr. C. Rüchardt für Anregungen.

\section{Literatur}

1. Langhals H (1980) Nachr Chem Tech Lab 28:716

2. Rademacher A, Märkle S, Langhals H (1982) Chem Ber 115:2927

3. Lukáč I, Langhals H (1983) Chem Ber 116:3524

4. Langhals H (1980) DE 3016764 A1 vom 30.4. 1980

5. Friedrich J, Haarer D (1984) Angew Chem 96:96 [Angew Chem Int Ed Engl (1984) 23:113]

6. Personov RE (1981) J Lumines 24/25:475

7. Aubert C (1984) Dissertation, Univ Basel

8. Rademacher A (1981) Dissertation, Univ Freiburg

Eingegangen am 8. Juni 1984 